\title{
Postoperative Management of Multiple Primary Cancers Associated with Non-small Cell Lung Cancer
}

\author{
FUMIHIRO SHOJI $^{1,2}$, KOJI YAMAZAKI ${ }^{1}$, NAOKO MIURA ${ }^{1}$, MASAKAZU KATSURA $^{1}$, \\ YUKA OKU $^{1}$, SADANORI TAKEO $^{1}$ and YOSHIHIKO MAEHARA ${ }^{2}$ \\ ${ }^{1}$ Department of Thoracic Surgery, Clinical Research Institute, \\ National Hospital Organization Kyushu Medical Center, Fukuoka, Japan; \\ ${ }^{2}$ Departement of Surgery and Science, Graduate School of Medical Sciences, Kyushu University, Fukuoka, Japan
}

\begin{abstract}
Background/Aim: Modern treatment for primary cancers has improved survival. Therefore, increased numbers of patients with multiple primary cancers (MPC) associated with lung cancer may be expected. The aim of the present study was to report MPC associated with lung cancer and discuss patients' characteristics and postoperative management. Patients and Methods: Overall, 973 consecutive patients who underwent surgery for non-small cell lung cancer (NSCLC) were retrospectively studied. Results: NSCLC with MPC was observed in 148 patients (15.2\%). MPC comprised 24 synchronous (2.5\%) and 124 metachronous (12.7\%) diseases. Of the 124 metachronous patients, NSCLC was detected before cancers were detected in other organs (lung cancer first $(L C F)$ ) in 25 (20.2\%) patients and subsequently in other organs after treatment (other organs, primary cancer-first $(O C F)$ ) in 99 (79.8\%) patients. MPC was significantly associated with advanced age $(p<0.0001)$ and chronic obstructive pulmonary disease $(C O P D)(p=0.0040)$. The leading sites of MPC in patients with synchronous tumors and those with $O C F$ were the digestive organs. In contrast, the leading site of MPC in patients with LCF was the lung. In the latter, at least two primary lung cancers were detected within 5 years as well as 5 years after surgery for the treatment of the first detected lung cancer, while primary cancers of other organs were detected within 5 years. Conclusion: Advanced age and COPD may represent a high-
\end{abstract}

Correspondence to: Fumihiro Shoji, MD, Ph.D., Department of Thoracic Surgery, Clinical Research Institute, National Hospital Organization Kyushu Medical Center, Fukuoka, Japan, 1-8-1, Jigyohama, Chuo-ku, Fukuoka, 810-8563, Japan. Tel: +81 928520700, Fax: +81 928468485, e-mail: fshoji@surg2.med.kyushuu.ac.jp

Key Words: Multiple primary cancers (MPC), non-small cell lung cancer (NSCLC), lung cancer first (LCF), other organs, primary cancer-first $(\mathrm{OCF})$, postoperative management. risk of MPCs. Therefore, we recommend careful follow-up to detect MPC in the lung as well as the digestive organs beyond 5 years after treatment of the first cancer.

Approximately $30 \%$ of the world's population will develop a malignancy, and approximately $10 \%$ will develop second malignancies (1). Although multiple malignancies may be caused by carcinogens, environmental factors, or hereditary factors (2), the cause of multiple malignancies is unknown. Lung cancer is the leading cause of cancer death in Japan and worldwide (3). For example, in Japan in 2009, >800,000 patients had primary cancers, and approximately 100,000 patients suffered from tracheal, bronchus, and lung cancers, the latter representing the leading type of primary cancers (4).

Modern treatment of primary cancers has improved survival. Therefore, increased numbers of patients with lung cancer with multiple primary cancers (MPC) may be expected. Numerous studies, that were conducted in the United States, Europe, and Asia, focused on MPC associated with lung cancer (2, 5-9). Thus, a structured screening program may be required to detect development of MPCs after surgery for lung cancer. To our knowledge, a comprehensive plan to improve survival for such patients is not available. Identification of the type of MPC and the period at risk may help thoracic surgeons and oncologists to design postoperative surveillance plans for patients with lung cancer. Therefore, the aim of this study was to clarify the frequency, type, and the detected period of MPC associated with lung cancer and discuss patients' clinical characteristics and postoperative management.

\section{Patients and Methods}

Patients. The Institutional Review Board of our hospital granted approval for this study (15C079). The charts of 973 consecutive patients who underwent surgery for NSCLC at the National Hospital Organization Kyushu Medical Center from July 1994 to December 2009 were reviewed. 
Table I. Synchronous and metachronous primary cancers and patients' characteristics.

\begin{tabular}{|c|c|c|c|c|c|}
\hline \multirow[b]{2}{*}{ Items } & \multirow[b]{2}{*}{ Total $(n=973)$} & \multicolumn{3}{|c|}{ Multiple primary cancer } & \multirow[b]{2}{*}{$p$-Value } \\
\hline & & None $(\mathrm{n}=825)$ & Synchronous $(\mathrm{n}=24)$ & Metachronous $(n=124)$ & \\
\hline Age $($ mean \pm SD) & $67.3 \pm 10.3$ & $66.5 \pm 10.8$ & $73.2 \pm 9.7$ & $71.0 \pm 9.3$ & $<0.0001$ \\
\hline Gender & & & & & 0.4485 \\
\hline Male & $626(64.3)$ & $525(63.6)$ & $15(62.5)$ & $86(69.4)$ & \\
\hline Female & $347(35.7)$ & $300(36.4)$ & $9(37.5)$ & $38(30.6)$ & \\
\hline Smoking history & & & & & 0.9015 \\
\hline Current/former & $649(66.7)$ & $550(66.7)$ & $17(70.8)$ & $82(66.1)$ & \\
\hline Never & $324(33.3)$ & $275(33.3)$ & $7(29.2)$ & $42(33.9)$ & \\
\hline ECOG PS & & & & & 0.2428 \\
\hline 0 & $802(82.4)$ & $683(82.8)$ & $19(79.2)$ & $100(80.7)$ & \\
\hline 1 & $138(14.2)$ & $111(13.4)$ & $5(20.8)$ & $22(17.7)$ & \\
\hline 2 & $33(3.4)$ & $31(3.8)$ & $0(0.0)$ & $2(1.6)$ & \\
\hline Respiratory comorbidities & & & & & 0.0040 \\
\hline Yes & $234(24.1)$ & $182(22.1)$ & $9(37.5)$ & $43(34.7)$ & \\
\hline No & 739 (75.9) & $643(77.9)$ & $15(62.5)$ & $81(65.3)$ & \\
\hline Year of operation & & & & & 0.8986 \\
\hline 1995-1999 & $184(18.9)$ & $155(18.8)$ & $4(16.7)$ & $25(20.2)$ & \\
\hline $2000-2009$ & $789(81.1)$ & $670(81.2)$ & $20(83.3)$ & $99(79.8)$ & \\
\hline Histology & & & & & 0.2042 \\
\hline Adenocarcinoma & $653(67.1)$ & $554(67.2)$ & $20(83.4)$ & 79 (63.7) & \\
\hline Squamous cell carcinoma & $248(25.5)$ & $209(25.3)$ & $2(8.3)$ & $37(29.8)$ & \\
\hline Others & $72(7.4)$ & $62(7.5)$ & $2(8.3)$ & $8(6.5)$ & \\
\hline Pathological stage & & & & & 0.2145 \\
\hline I & $594(61.0)$ & $490(59.4)$ & $19(79.2)$ & $85(68.6)$ & \\
\hline II & $181(18.6)$ & $159(19.3)$ & $3(12.5)$ & $19(15.3)$ & \\
\hline III & $178(18.3)$ & $158(19.1)$ & $2(8.3)$ & $18(14.5)$ & \\
\hline IV & $20(2.1)$ & $18(2.2)$ & $0(0.0)$ & $2(1.6)$ & \\
\hline Surgical procedures & & & & & $<0.0001$ \\
\hline Pneumonectomy & $27(2.7)$ & $25(3.0)$ & $0(0.0)$ & $2(1.6)$ & \\
\hline Lobectomy & $785(80.7)$ & $686(83.2)$ & $17(70.8)$ & $82(66.1)$ & \\
\hline Sublobar resections & $161(16.6)$ & $114(13.8)$ & $7(29.2)$ & $40(32.3)$ & \\
\hline
\end{tabular}

PS: Performance status; SD: standard deviation.

Data collection. Data included patients' clinical characteristics and lung cancer diagnosis, cancer stage, pathological type, lung cancer treatment, and survival. This study included 626 men and 347 women, ranging from 20 years to 91 years (median 69 years) with malignancies of other organs. The results were determined in followup examinations conducted from of 0 to 208.8 months (median 47.8 months). Blood tests, including tumor markers, and chest X-rays, were evaluated at 3- or 4-month intervals during the first year and at 6-month intervals thereafter. Postoperative follow-up (6-month intervals during the first year and yearly thereafter) comprised chest computed tomography (CT), abdominal CT, bone scintigraphy, brain magnetic resonance imaging, or positron emission tomography. Data included clinical characteristics, date of diagnosis of cancer of the lungs and other organs, cancer stage, pathological type, lung cancer treatment, survival, recurrence, and prognosis. Pathologic staging of primary lung cancer was undertaken according to the TNM Classification of Malignant Tumors, 7th edition (10).

Definition of MPC. MPC is a term that presents a definite picture of multiple malignancies with the possibility of including a metastasis or excluding a recurrence (11). Further, according to the timing of the detection of a separate malignancy, MPC addresses a synchronous or metachronous malignancy. Moertel et al. (12) define synchronous malignancies as those that occur within 6 months following the diagnosis of a previous cancer. In contrast, metachronous malignancies may appear more than 6 months apart. Multiple independent primary lung cancers are defined by the Martini and Melamed criteria (13). Moreover, metachronous lung cancers were divided into those detected before those in other organs (lung cancer first-group; LCF group) and after detection of primary cancers of other organs (other organs, primary cancer-first group, OCF group).

Statistical analysis. Statistical analyses of categorical factors were performed using Fisher's exact tests. Overall survival (OS) was defined as the period between surgery and the date of the last followup or death. These rates were estimated using the Kaplan-Meier method with the log-rank test. Cox proportional hazards analysis was performed to estimate the hazard ratios for positive risk factors associated with the incidence of metachronous primary cancers after lung cancer. All statistical analyses were performed using JMP Statistical Discovery Software (version 13.0; SAS Institute, Cary, NC, USA). Results were considered statistically significant if $p<0.05$. 


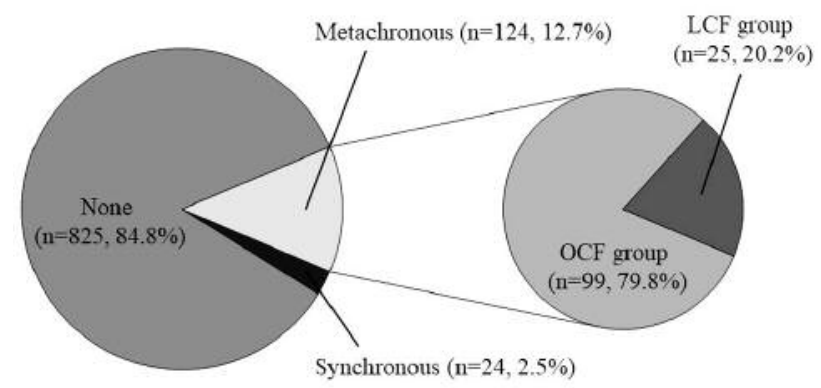

Figure 1. Distribution of patients according to the detection periods of lung cancer and multiple primary cancers of other organs. OCF: Other organs cancer first detected; LCF: lung cancer first detected.

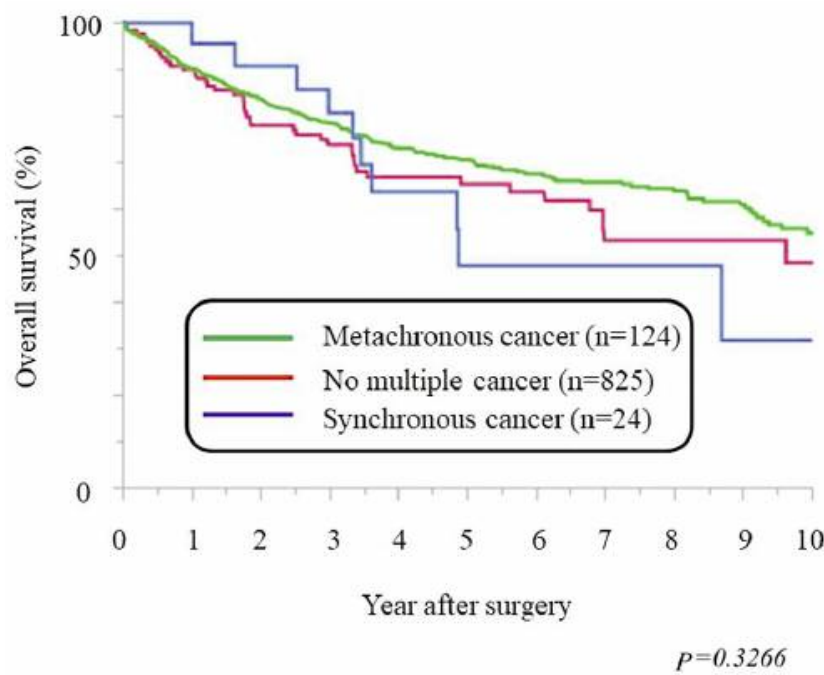

Figure 2. Kaplan-Meier curves showing survival vs. multiple cancers of other organs.

\section{Results}

Frequency of lung cancer in patients with MPC. As shown in Figure 1, lung cancer with MPC was observed in 148 patients $(15.2 \%)$. MPCs comprised 24 synchronous $(2.5 \%)$ and 124 metachronous $(12.7 \%)$ cancers. In metachronous $25 / 124(20.2 \%)$ patients, lung cancer was detected before cancers of other organs (LCF group) and subsequent to cancers of other organs following treatment in 99 (79.8\%) patients (OCF group). Triple primary cancers were identified in four synchronous patients. In the OCF group, three and four primary cancers were identified in 10 and one patient, respectively. In the LCF group, three and six primary cancers were identified in five and one patient, respectively.
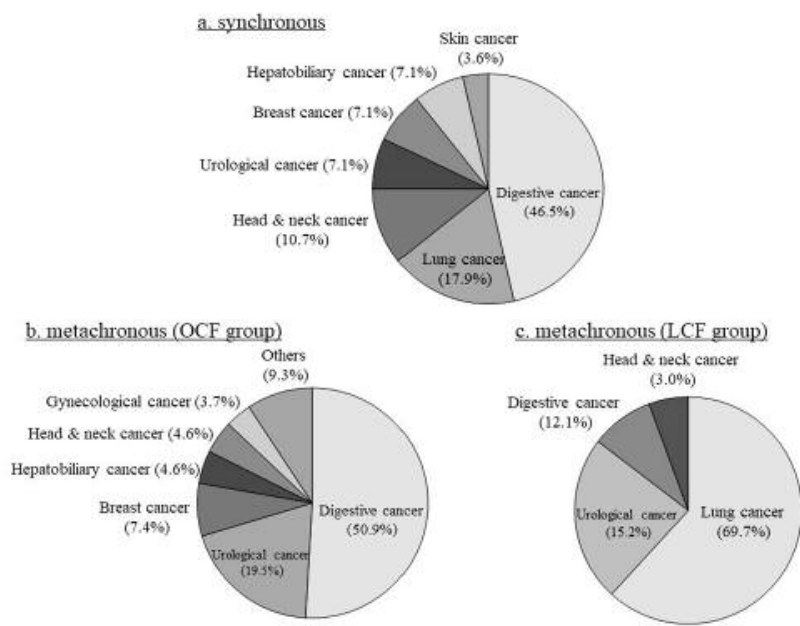

Figure 3. Distribution of multiple primary cancers of other organs in patients with synchronous and metachronous lung cancer. A, synchronous patients; $B$, metachronous patients $(O C F)$ and $C$, metachronous patients ( $L C F)$.

Clinicopathological features of patients with MPC. Table I presents the associations between MPC and various clinicopathological features. MPC was significantly associated with age $(p<0.0001)$, respiratory comorbidities $(p=0.0040)$, and surgery $(p<0.0001)$, although no significant association was identified between MPC and sex, smoking history, ECOG PS, year of surgery, histology, or pathological stage. Table II presents the details of respiratory comorbidities. The five leading respiratory comorbidities in 234 patients were chronic obstructive respiratory disease (COPD) (41.4\%), old tuberculosis (23.1\%), interstitial pneumonia (IP) $(14.9 \%)$, bronchial asthma $(11.1 \%)$, and idiopathic pulmonary fibrosis (IPF) $(7.4 \%)$.

Overall survival of patients with MPC. Kaplan-Meier curves depicting OS as a function of MPC are shown in Figure 2. There was no statistically significant difference among the groups without multiple cancers, synchronous cancers, or metachronous cancers (log-rank, $p=0.3266)$.

Details of $M P C$. Figure 3 shows the distribution of patients with MPCs. In synchronous cases, the leading three sites were digestive cancers $(46.5 \%)$, lung cancer $(17.9 \%)$, and head and neck cancer $(10.7 \%)$. In metachronous cases in the OCF group, the leading three sites were digestive cancers $(50.9 \%)$, urological cancer $(19.5 \%)$, and breast cancer (7.4\%). In contrast, in the LCF group, the leading three sites were lung cancer $(69.7 \%)$, urological cancer $(15.2 \%)$. and digestive cancer $(12.1 \%)$. 


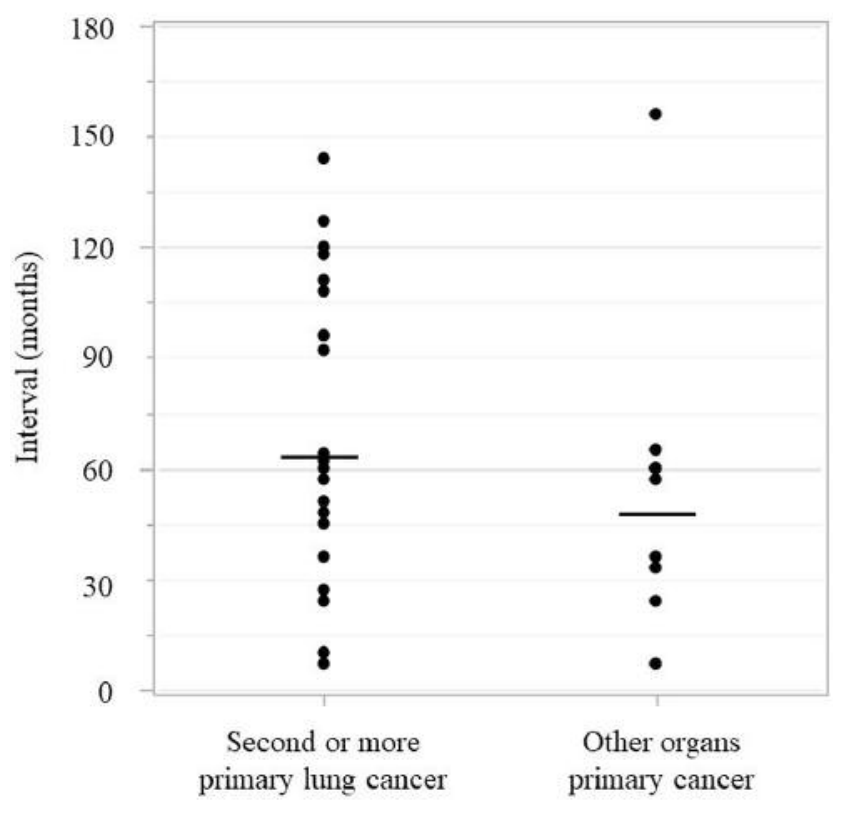

Figure 4. Time between the date of diagnosis of lung cancer and that of multiple primary cancers of other organs in LCF patients.

Detection of MPC in the LCF group. Figure 4 shows the interval between the detection of lung cancer and that of primary cancers of other organs in the LCF group. In this group, most primary cancers of other organs were detected within 5 years after detection of the first lung cancer (average, 46.5 months). In contrast, at least two primary lung cancers were detected 5 years after detection of the first lung cancer (average, 62 months).

Correlation of respiratory comorbidities and MPC in LCF group. A forest plot of odds ratios (OR) of the risk factors COPD, IP, and IPF for MPC of the LCF group is shown in Figure 5. Patients with respiratory comorbidities had a 2-fold higher prevalence of MPC (OR 2.00, 95\% confidence interval 1.60 to $7.92, p=0.0018$ ) compared with those without respiratory comorbidities. Patients with COPD had a 5-fold higher prevalence of MPC (OR 5.25, 95\% confidence interval $2.20-12.55, p=0.0002)$.

\section{Discussion}

Recent advances in less-invasive surgery, anesthesia, and perioperative management enable patients with lung cancer with preoperative comorbidities to achieve acceptable longterm survival (14). Because of carcinogens, environmental or hereditary factors, patients with a history of lung cancer are at risk for MPC $(2,7)$. Thus, it is necessary to follow lung cancer patients adequately to detect MPC.

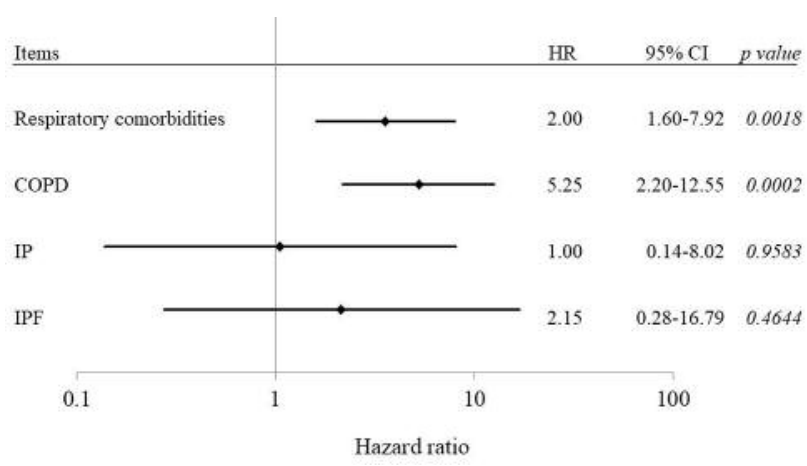

Figure 5. Forest plot of hazard ratios (HR) for risk factors for patients with multiple primary cancers in the LCF group. COPD: Chronic obstructive pulmonary disease; IP: interstitial pneumonia; IPF: idiopathic pulmonary fibrosis; 95\% CI: 95\% confidence interval.

Table II. Respiratory comorbidities (overlapping).

\begin{tabular}{lc}
\hline Items & Number of patients $(\%)$ \\
\hline Number of patients & $234(100)$ \\
Chronic obstructive pulmonary disease & $97(41.4)$ \\
Old tuberculosis & $54(23.1)$ \\
Interstitial pneumonia & $35(14.9)$ \\
Bronchial asthma & $26(11.1)$ \\
Idiopathic pulmonary fibrosis & $17(7.3)$ \\
Congenial cystic disease & $8(3.4)$ \\
Others & $54(23.0)$ \\
\hline
\end{tabular}

The emphasis of the present study was on the following points: First, the frequency of MPC was $15.2 \%$ in lung cancer patients who underwent surgery. The percentage of patients with MPC associated with lung cancer has recently increased, and several studies report similar percentages $(>10 \%)$ of MPC $(8,15)$. Generally, patients with metachronous cancer have a better prognosis compared with those with synchronous tumors because of careful postoperative followup of the initially detected cancer (6). Moreover, Aguiló et al. (8) found that multiple cancers are not significantly associated with worse prognosis. Similarly, our present results do not indicate a significant difference between the prognosis of patients without MPC, metachronous cancer, or synchronous cancer. Therefore, thorough screening designed to diagnose MPC, at the earliest, may lead to increased longterm survival of these patients.

Second, advanced age and respiratory comorbidities, mainly COPD and sublobar resection for lung cancer, were risk factors for the occurrence of MPC. Further, our subgroup analysis reveals that patients with LCF with COPD 
had a 5-fold higher prevalence of MPC compared with those without COPD. There is a close association between COPD and the development of lung cancer (16), and smoking is the most important independent risk factor of cancer multiplicity $(5,8,9,17)$. Although patients with COPD patients had a history of smoking, our data reveal that only COPD was a risk factor for MPC.

This result may be explained as follows: Among the 97 patients with COPD studied here, the amount of tobacco use of 86 patients $(88.7 \%)$ according to the Brinkman Index (BI) was analyzed. The BI of $71 / 86$ patients $(82.6 \%)$ was $>400$, indicating heavy smoking. Thus, COPD was likely the result of heavy smoking, indicating that this habit is a risk factor for MPC. In contrast, an emphysematous change may not always be recognized in patients with a history of smoking. Henschke et al. (18) found CT findings of emphysema in never smokers and that their emphysema could be caused by secondhand tobacco smoke. Therefore, never-smoking LCF patients who exhibit emphysematous changes should be carefully checked and CT should be used to detect MPC.

The identification of sublobar resection as a risk factor for MPC in the present study may be explained by the selection of patients with early-staged lung cancer who underwent this treatment. Moreover, 143/161 patients (88.8\%) were diagnosed with pathological stage I NSCLC. Therefore, MPC may occur in long-term survivors of early-stage NSCLC, which supports the findings of a previous study, which shows that the probability of developing a second cancer depends on surviving a first cancer (8).

Third, the leading sites of MPC in synchronous and patients with OCF were digestive organs, consistent with findings from previous studies (5). Li et al. (6) found that lung cancer in Korean subjects is closely associated with digestive cancers because of smoking and that the most frequent sites (44.0\%) of MPC are digestive organs such as the stomach and colorectum (15). In contrast, in this study the leading site of MPC in patients with LCF was the lung, which is consistent with previous reports $(2,5,19)$. Thus, it is important to recognize that the major site of MPC differs between patients with OCF or LCF. Therefore, surgeons and oncologists should pay special attention to the incidence of cancers of digestive organs and the lung to optimize postoperative management.

Fourth, in patients with LCF, at least two primary lung cancers were detected within the first 5 years after surgery to treat the first detected lung cancer and thereafter, while primary cancers of other organs were detected within 5 years. Liu et al. (5) found that $>50 \%$ of patients had multiple primary cancers within 1 year of diagnosis of the first lung cancer, and $83.4 \%$ of the patients experienced an occurrence within 5 years. Duchateau et al. (2) found that approximately $80 \%$ of second primary cancers identified after NSCLC are diagnosed within the first year. Reinmuth et al. (20) found that leading site of MPC in patients with LCF is the lung
(56.2\%), and the median interval between diagnosis of the first and secondary lung cancers is 72 months. Surapaneni et al. (7) found that the risk of developing a second lung cancer is highest during the first year and continues to occur at a high frequency 10 years after treatment of the first lung cancer. Further, there is an increased risk of gastrointestinal cancers such as esophageal cancer, gastric cancer, and colorectal cancer after the diagnosis of lung cancer during the first few years, which gradually decreases to that of the general population because of risk mitigation, including smoking cessation and occupational changes (7). These results are consistent with our present findings.

The National Comprehensive Cancer Network's guidelines recommend conducting surveillance for NSCLC as follows: medical history, physical examination (H\&P), and chest CT every 6 to 12 months for the first 2 years, followed by annual H\&P and chest CT. In the present study, the leading site of metachronous primary cancer was the lung; however, other organs were not excluded as metachronous sites. Our data indicate the requirement for careful long-term follow up, and it may be necessary to establish a thorough postoperative screening plan to examine the lungs as well as other organs.

Our retrospective results are valuable and informative for surgeons and oncologists. However, a limitation of the present study was that it was a retrospective study of patients treated at a single institution. Thus, large and multicenter clinical trials using adequate postoperative screening plans designed to detect MPC will be required.

In conclusion, a series of MPC were found to be associated with lung cancer. Patients of advanced age, those with COPD, or those who underwent sublobar resection for lung cancer may represent high-risk groups for MPC. Therefore, careful follow-up is recommended to detect MPC of the lung and other organs even as long as 5 years after treatment of the first cancer.

\section{Conflicts of Interest}

All Authors disclose no financial and personal relationships with other people or organizations that could inappropriately influence their work.

\section{Acknowledgements}

The Authors thank Edanz Group (www.edanzediting.com) for editing a draft of this manuscript.

\section{References}

1 Rheingold SR, Neugut AI and Meadows AT: Second cancers: incidence, risk factors, and management. In: Cancer Medicine. Bast RC, Kufe DW, Pollock RE, et al., (eds.). Hamilton, ON, Canada. BC: Decker, pp. 2399-2406, 2000. 
2 Duchateau CS and Stokkel MP: Second primary tumors involving non-small cell lung cancer: prevalence and its influence on survival. Chest 127: 1152-1158, 2005.

3 Siegel RL, Miller KD and Jemal A: Cancer statistics, 2017. CA Cancer J Clin 67: 7-30, 2017.

4 Hori M, Matsuda T, Shibata A, Katanoda K, Sobue T and Nishimoto H: Japan Cancer Surveillance Research Group. Cancer incidence and incidence rates in Japan in 2009: a study of 32 population-based cancer registries for the Monitoring of Cancer Incidence in Japan (MCIJ) project. Jpn J Clin Oncol 45: 884-891, 2015.

5 Liu YY, Chen YM, Yen SH, Tsai CM and Perng RP: Multiple primary malignancies involving lung cancer-clinical characteristics and prognosis. Lung Cancer 35: 189-194, 2002.

6 Li F, Zhong WZ, Niu FY, Zhao N, Yang JJ, Yan HH and Wu YL: Multiple primary malignancies involving lung cancer. BMC Cancer 15: 696, 2015.

7 Surapaneni R, Singh P, Rajagopalan K and Hageboutros A: Stage I lung cancer survivorship: risk of second malignancies and need for individualized care plan. J Thorac Oncol 7: 12521256, 2012.

8 Aguiló R, Macià F, Porta M, Casamitjana M, Minguella J and Novoa AM: Multiple independent primary cancers do not adversely affect survival of the lung cancer patient. Eur $\mathbf{J}$ Cardiothorac Surg 34: 1075-1080, 2008.

9 Romaszko A, Świetlik E, Doboszyńska A, Szpruch P and Luks $\mathrm{J}$ : Lung cancer and multiple neoplasms: a retrospective analysis. Adv Exp Med Biol 911: 53-58, 2016.

10 Travis WD, Giroux DJ, Chansky K, Crowley J, Asamura H, Brambilla E, Jett J, Kennedy C, Rami-Porta R, Rusch VW and Goldstraw P; International Staging Committee and Participating Institutions: The IASLC Lung Cancer Staging Project: proposals for the inclusion of broncho-pulmonary carcinoid tumors in the forthcoming (seventh) edition of the TNM Classification for Lung Cancer. J Thorac Oncol 3: 1213-1223, 2008.

11 Warren S and Gates O: Multiple primary malignant tumors: a survey of the literature and a statistical study. Am J Cancer 16: 1358-1414, 1932.

12 Moertel CG, Bargen JA and Dockerty MB: Multiple carcinomas of the large intestine: a review of the literature and a study of 261 cases. Gastroenterology 34: 85-98, 1958.
13 Martini N and Melamed MR: Multiple primary lung cancers. J Thorac Cardiovasc Surg 70: 606-612, 1975.

14 Brock MV, Kim MP, Hooker CM, Alberg AJ, Jordan MM, Roig $\mathrm{CM}, \mathrm{Xu} \mathrm{L}$ and Yang SC: Pulmonary resection in octogenarians with stage I non-small cell lung cancer: a 22-year experience. Ann Thorac Surg 77: 271-277, 2004.

15 Son C, Lee SK, Choi PJ and Roh MS: Characteristics of additional primary malignancies in Korean patients with nonsmall cell lung cancer. J Thorac Dis 5: 737-744, 2013.

16 Dai J, Yang P, Cox A and Jiang G: Lung cancer and chronic obstructive pulmonary disease: From a clinical perspective. Oncotarget 8: 18513-18524, 2017.

17 Shiels MS, Gibson T, Sampson J, Albanes D, Andreotti G, Beane Freeman L, Berrington de Gonzalez A, Caporaso N, Curtis RE, Elena J, Freedman ND, Robien K, Black A and Morton LM: Cigarette smoking prior to first cancer and risk of second smoking-associated cancers among survivors of bladder, kidney, head and neck, and stage I lung cancers. J Clin Oncol 32: 39893995, 2014

18 Henschke CI, Yip R, Boffetta P, Markowitz S, Miller A, Hanaoka T, Wu N, Zulueta JJ and Yankelevitz DF; I-ELCAP Investigators: CT screening for lung cancer: Importance of emphysema for never smokers and smokers. Lung Cancer 88: 42-47, 2015.

19 Duchateau CS and Stokkel MP: Second primary tumors involving non-small cell lung cancer: prevalence and its influence on survival. Chest 127: 1152-1158, 2005.

20 Reinmuth N, Stumpf A, Stumpf P, Muley T, Kobinger S, Hoffmann H, Herth FJ, Schnabel PA, Warth A, Bischoff $\mathrm{H}$ and Thomas M: Characteristics and outcome of patients with second primary lung cancer. Eur Respir J 42: 1668-1676, 2013. 